Respected editor in chief, JBCPS,

I am a regular reader of the articles published in your journal and happy observing the gradual progress of the Journal.

Narrative review day by day is losing it weightage as a research publication and simultaneously the systematic review is becoming more popular in the process of summarizing evidence during the last couple of decades. I personally feel that the young contributors of the journal should be encouraged to be involved in this field of research. I will feel honoured, if I can contribute in any way in the motivation process.

With this letter I am sending a summary write up on systematic review writing with the title 'Systematic Review: Requirement of the Time'. Hope this will be able to create interest in the junior researcher to be involved in this field. I feel honoured, thereby.

Wishing more contributory progress to your journal.

Brig Gen (Retd) Md Ahsan Habib, MBBS, M Phil, M MEd

Professor and Medical Educationist

Head, Department of Anatomy, International Medical College

Visiting Professor, BIRDEM Academy and BSMMU

\title{
Systematic Review: Requirement of the Time
}

\section{MA HABIB}

\section{Introduction:}

Evidence-based medical education (Evidence-based medicine) is a well discussed approach to the teaching and practice of medicine during the last two decades ${ }^{1}$. In this recent COVID-19 situation the influence of evidence-based medicine in patient care and use of healthcare resources worldwide has become more evident ${ }^{2}$. It is very difficult for clinicians, health care Stakeholders and Associates to extract admirable aspects from the bulk of primary research which is growing at an ever-increasing rate. The excellence in patient care correlates with the use of the best currently available evidence. In order to access and utilize that research information, the physicians require a unique set of knowledge and skills which are not part of traditional medical education.

Review of original research work on a particular topic or question can inform an up-to-date and complete understanding with evidence and lead to implementation of the agenda for evidence-based medicine. Over the last half a century various institute and organizations are working on traditional narrative review to make it more representative as well as bias free ${ }^{3-6}$. Mentionable contribution has been made by Cochrane Collaboration group and PRISMA statement to develop a systematic review guideline. Various aspects of systematic review methodology are available now, including:

o Searching for studies

o Statistics and meta-analysis (MA) including prospective MA and individual participant data MA

o Assessing bias

o Use of non-randomized designs

o The incorporation of qualitative and economic data

o The applicability and interpretation of the findings of systematic reviews

o Patient reported outcomes, equity issues, screening and diagnostic tests, and prognosis

Systematic reviews and meta-analyses have become the gold standard of research reviews. Systematic review methods can be applied to almost any study type and aim to be systematic, explicit and reproducible and metaanalysis methods are used to examine the research findings (specifically strength of the relationship).

\section{Historical Background}

Archie Cochrane first clearly expressed the need for applied scientific evidence over the expert opinion of 
clinicians and published a book on systematic review in medical science ${ }^{3}$. He developed a web-based Cochrane Collaboration Group, which is working during last half a century to produce an acceptable systematic review methodology and to set of priorities for systematic reviews and other research ${ }^{4}$.

Subsequently several groups developed to address the suboptimal review and meta-analyses and tried to suggest guideline to standardize the process. Of those QUOROM Statement (Quality of Reporting of Metaanalyses) ${ }^{5}$ and PRISMA (Preferred Reporting Items for Systematic reviews and Meta-Analyses) $)^{6}$ could attract the researcher, who are interested to work in this field, where the first one was to improving the quality of reporting of meta-analyses of clinical randomized controlled trials (RCTs). The PRISMA statement covered both systematic reviews and meta-analyses. Both these statements have proposed check list and flow diagram to make the process flawless, smooth and uniform.

Selection of Topic or Question for Systematic review and Searching Tool

Decision on review topic, formulation of question for that topic and structuring that to a purposive internet searches are very critical job. This is the primary requirement of selection of desired research papers for a systematic review in an unbiased way. Various tools are developed by researcher like PICO (Population/ problem, Intervention/exposure, Comparison, Outcomes), SPIDER (Sample, Phenomenon of Interest, Design, Evaluation, Research type), SPICE (Setting, Population or Perspective, Intervention, Comparison, Evaluation), RETREAT Mnemonic (Review Question, Epistemology, Time, Resources, Expertise, Audience and Purpose, Type of Data), ECLIPSE (Expectation, Client group, Location, Impact, Professionals, Service) and CIMO (Context, Intervention, Mechanism, Outcome) ${ }^{7-}$ 9 . These are effective in developing inclusion and exclusion criteria for selection of a systematic review as well. Through this strenuous job when a researcher arrives at a decision to conduct a review research in an acceptable way PRISMA 2020 statement is a pretty good, comfortable and more complete guideline to achieve the goal ${ }^{6}$. Events of that process (writing systematic review) are described below in brief.

\section{Title writing}

The title should contain key information about the main objective or question. Inclusion of 'systematic review' in the title facilitates identification by potential users or mentioning it as 'a systematic review with meta-analysis' will give additional information as meta-analysis refers only to the statistical synthesis. Inclusion of design of the reviewed research papers like 'a systematic review of randomized trials' will carry more clear information to readers. The terms like 'review', 'literature review', 'evidence synthesis' and 'knowledge synthesis' are not recommended.

\section{Abstract Writing}

An abstract should provide key information about all the components like main objective(s) that the review has addressed, methodology followed, salient findings and impacts of the findings, so that a reader can decide whether to access the full report.

\section{Writing Introduction}

Describing rationale should be the main target. The readers should get an idea about the existing knowledge situation and the reason of this review. Author should express expectation of valuable addition to the existing knowledge. At the end the reader should get an explicit statement of the objective(s) or question(s) the review has addressed.

\section{Method Writing}

The method should present full paper searching strategy mentioning the target sources (databases, registers and websites) and time of searching. Inclusion and exclusion criteria should be fixed justifiably (vide supra). Steps followed to retrieve the sources and specific reasons for exclusion are encouraged to keep in recorded form. Search strategy and selection procedure should be reproducible. Detail description of procedure of information extraction (qualitative as well as quantitative) and all outcomes for which data were sought should be defined. Effect measures (e.g. risk ratio, mean difference) are statistical constructs that compare outcome data between two groups and should be specifically mentioned. Method should contain the process used to decide and to prepare the data for presentation as well as for the sake of result synthesis.

\section{Result Synthesis and Writing}

Results of the search and selection process, from the sources selected in method can be presented in a flow chart as proposed by PRISMA statement- $2020^{6}$ in a summarized form. This will help the readers to understand procedure and result of search and selection process. Description of characteristics of the studies included in review to allow readers to understand the applicability of the review is essential. The summary statistics, effect estimate and its precision (such as 
confidence/credible interval) of all outcomes should be presented in suitable forms of presentation. If worthy and researcher is interested, meta-analysis can be done. In that case, presentation for each the summary estimate, its precision (such as confidence/credible interval) and heterogeneity measures of statistical analysis are required.

\section{Discussion Part}

The discussion should provide a general interpretation of the results in the context of other evidences, limitations of the evidence included in the review, limitations of the review processes used and implications of the results for practice, policy and future research. The readers may get idea on results of other similar systematic reviews with comparison. The reviewer may have access limitations, language limitation and regional fascination. Author's way of expression on implication of findings may attract patients and healthcare providers or policy makers and administrators. At the end author may recommend further investigation in light of experience gained during the process.

\section{References:}

1. Alan S. Evidence-Based Medicine: A New Paradigm for the Teaching and Practice of Medicine. Annals of Saudi Medicine 1999, 19(1) : 32-36. (https://www.annsaudimed.net/doi/ 10.5144/0256-4947.1999.32)

2. McManus NM, Offman R, Oetman JD. Emergency Department Management of COVID-19: An Evidence-Based Approach. West J Emerg Med. 2020 Sep 25;21(6):32-44. doi: 10.5811/westjem.2020.8.48288. PMID: 33052814; PMCID: PMC7673887.

3. Cochrane AL. Effectiveness and efficiency Random Reflection on Health Service. The Nuffield Provincial Hospital Trust : UK; 1972.

4. Higgins JPT, Thomas J, eds. Cochrane Handbook for Systematic Reviews of Interventions. $2^{\text {nd }}$ Edn. John Wiley \& Sons, Inc : USA; 2019.

5. Moher D, Cook DJ, Eastwood S, Olkin I, Rennie D, Stroup DF. Improving the quality of reports of meta-analyses of randomised controlled trials: the QUOROM statement. Quality of Reporting of Meta-analyses.Lancet. 1999 Nov 27;354(9193):1896-900. doi: 10.1016/s01406736(99)04149-5. PMID: 10584742.

6. Page M J, Moher D, Bossuyt P M, Boutron I, Hoffmann T C, Mulrow C D et al. PRISMA 2020 explanation and elaboration: updated guidance and exemplars for reporting systematic reviews BMJ 2021; 372 :n160. doi:10.1136/ bmj.n 160

7. Cooke A, Smith D, Booth A. Beyond PICO: The SPIDER Tool for Qualitative Evidence Synthesis. Qualitative Health Research. 2012;22(10):1435-1443. doi:10.1177/ 1049732312452938

8. Booth A. Formulating questions to explore complex interventions within qualitative evidence synthesis. Library Hi Tech2006, 24(3): 355-368 doi 10.1108/073788306 10692127

9. Booth A, Noyes J, Flemming K, Moore G, TunçalpO, Shakibazadeh E. Formulating questions to explore complex interventions within qualitative evidence synthesis. BMJ Glob Health 2019;4:e001107. doi:10.1136/bmjgh-2018-001107 